\title{
Exosome-transmitted long non-coding RNA PTENP1 suppresses bladder cancer progression
}

\author{
Rui Zheng ${ }^{1,2 \dagger}$, Mulong Du ${ }^{1,2,3 \dagger}$, Xiaowei Wang ${ }^{1,2 \dagger}$, Weidong $X u^{4 \dagger}$, Jiayuan Liang ${ }^{1,2}$, Wenying Wang ${ }^{5}$, Qiang Lv ${ }^{6}$, \\ Chao Qin ${ }^{6}$, Haiyan Chu ${ }^{1,2}$, Meilin Wang ${ }^{1,2}$, Lin Yuan ${ }^{7,8^{*}}$, Jing Qian ${ }^{9^{*}}$ and Zhengdong Zhang ${ }^{1,2^{*}}$
}

\begin{abstract}
Background: Extracellular communication within the tumor microenvironment plays a critical role in tumor progression. Although exosomes can package into long non-coding RNAs (IncRNAs) to mediate extracellular communication, the role of exosomal IncRNA PTENP1 in bladder cancer (BC) remains unclear.

Method: We detected PTENP1 expression between patients with BC and healthy controls; the expression occurred in tissues and exosomes from plasma. We assessed the diagnostic accuracy by the receiver operating characteristic curve (ROC) and the area under curve (AUC). Cell phenotypes and animal experiments were performed to determine the effect of exosomal PTENP1.

Results: PTENP1 was significantly reduced in $B C$ tissues and in exosomes from plasma of patients with $B C(P<0.05)$. We found that PTENP1 was mainly wrapped by exosomes. Exosomal PTENP1 could distinguish patients with BC from healthy controls (AUC $=0.743 ; 95 \%$ confidence interval $(C l)=0.645-0.840)$. Normal cells secreted exosomal PTENP1 and transmitted it to $B C$ cells, thus inhibiting the biological malignant behavior of $B C$ cells by increasing cell apoptosis and reducing the ability to invade and migrate $(P<0.05)$. Exosomal PTENP1 could suppress tumor growth in vivo. Furthermore, exosomal PTENP1 mediated the expression of PTEN by competitively binding to microRNA-17.

Conclusion: Exosomal PTENP1 is a promising novel biomarker that can be used for the clinical detection of BC. Exosomes derived from normal cells transfer PTENP1 to BC cells, which reduce the progression of BC both in vitro and in vivo and suggest that exosomal PTENP1 participates in normal-cell-to-bladder-cell communication during the carcinogenesis of BC.
\end{abstract}

Keywords: Bladder cancer, PTENP1, Exosomes, Biomarker, Progression

\section{Background}

Bladder cancer is one of the most prevalent malignancies of the urinary system $[1,2]$. The American Cancer Society estimated that there were 79,030 new cases of bladder cancer and 16,870 deaths due to bladder cancer last

\footnotetext{
* Correspondence: yuanlin47@126.com; qianjing998@163.com; drzdzhang@gmail.com

${ }^{\dagger}$ Rui Zheng, Mulong Du, Xiaowei Wang and Weidong Xu contributed equally to this work.

7 Department of Urology, Jiangsu Province Hospital of TCM, 155 Hanzhong Road, Nanjing 210029, People's Republic of China

${ }^{9}$ Department of General Surgery, Yizheng Hospital, Drum Tower Hospital Group of Nanjing, 1 Huannan Road, Yizheng 211900, People's Republic of China ${ }^{1}$ Department of Environmental Genomics, School of Public Health, Jiangsu Key Laboratory of Cancer Biomarkers, Prevention and Treatment, Collaborative Innovation Center for Cancer Personalized Medicine, Nanjing Medical University, 101 Longmian Avenue, Jiangning District, Nanjing 211166, China

Full list of author information is available at the end of the article
}

year in the United States [3]. In China, the incidence and mortality rates of bladder cancer have gradually increased in recent years $[4,5]$. Despite the improvement of cystoscopy, the prognosis of patients remains poor. Hence, these limitations prompted us to find new diagnostic indicators and therapeutic targets to improve the clinical efficacy of treatment for patients with bladder cancer.

Evidence has revealed that long non-coding RNAs (lncRNAs) are abnormally expressed in human cancers, including bladder cancer [6, 7]. LncRNAs are more than 200 nucleotides long with limited protein-encoding potential $[8,9]$, and contribute to the development of cancers by regulating several cellular processes that are crucial to tumorigenesis, such as cell proliferation, invasion, migration and apoptosis

(c) The Author(s). 2018 Open Access This article is distributed under the terms of the Creative Commons Attribution 4.0 International License (http://creativecommons.org/licenses/by/4.0/), which permits unrestricted use, distribution, and 
$[10,11]$. Exosomes are extracellular vesicles with endocytic origin that are secreted by most cell types. They are considered as extracellular messengers between tumor cells and their microenvironment by transmitting and exchanging their diverse cargoes, which includes lncRNAs [12-15]. Additionally, exosomal lncRNAs as clinical biomarkers are stable in blood and have the capacity to distinguish whether an individual has tumors or is healthy $[16,17]$.

In the present study, we selected 12 lncRNAs involved in the development of multiple tumors, including $H 19$, SNHG16, TUG1, UBC1, UCA1, MALAT1, MEG3, GAS5, ANRIL, HOTAIR, Kcnq1ot and PTENP1, and detected their expression both in tissues and exosomes of plasma. Our results revealed that PTENP1 was significantly decreased both in bladder cancer tissues and exosomes from bladder cancer plasma. PTENP1 is one of the pseudogene-expressed lncRNAs that plays a pivotal role in carcinogenesis $[18,19]$. Nevertheless, no data are currently available regarding the biological roles of exosomal PTENP1 in bladder cancer. The purpose of this study was to find a potential biomarker that could be used in the diagnosis of bladder cancer, and investigate if exosomal PTENP1 intervenes in cell-cell communication, which may result in the progression of bladder cancer.

\section{Methods \\ Study design and subjects}

All subjects gave written informed consent and this study protocol was approved by the institutional review board of Nanjing Medical University. This study included analysis of plasma samples from 50 patients with bladder cancer and 60 healthy controls, as well as 20 paired tumor and adjacent normal tissues, which were obtained from patients with bladder cancer from the First Affiliated Hospital of Nanjing Medical University and Jiangsu Province Hospital of Traditional Chinese Medicine.

\section{Bladder cancer cell lines}

Two bladder cancer cell lines (EJ and J82) and one normal human cell line (HEK 293A) were maintained under $5 \% \mathrm{CO}_{2}$ at $37{ }^{\circ} \mathrm{C}$ in RPMI-1640 medium (Gibco BRL, Rockville, Maryland, USA) with $10 \%$ fetal bovine serum (FBS, Gibco BRL).

\section{Exosome isolation}

The plasma and culture medium were collected and centrifuged at $3000 \mathrm{~g}$ for $15 \mathrm{~min}$ to remove cells and cellular debris. Exosomes were isolated using the Exoquick exosome precipitation solution (System Biosciences).
The details of exosome isolation are shown in the Additional file 1.

\section{Transmission electron microscopy (TEM)}

Exosomes were suspended in $100 \mu \mathrm{l}$ of PBS and were fixed with $5 \%$ glutaraldehyde at incubation temperature and then maintained at $4{ }^{\circ} \mathrm{C}$ until TEM analysis. According to the TEM sample preparation procedure, we placed a drop of exosome sample on a carbon-coated copper grid and immersed it in $2 \%$ phosphotungstic acid solution $(\mathrm{pH} 7.0)$ for $30 \mathrm{~s}$. The preparations were observed with a transmission electron microscope (Tecnai G2 Spirit Bio TWIN, FEI, USA).

\section{Western blots}

Protein were prepared with a detergent buffer, and the protein concentration was determined using the bicinchoninic acid (BCA) protein assay (Beyotime Institute of Biotechnology, Shanghai, China). Equal amounts $(60 \mu \mathrm{g})$ of protein samples were separated by a $12 \%$ gel using sodium dodecyl sulfate-polyacrylamide gel electrophoresis (SDS-PAGE) and transferred onto PVDF membranes (Millipore, Billerica, MA, USA). Monoclonal rabbit anti-TSG101 (ab125011, Abcam), monoclonal rabbit anti-CD63 (ab134045, Abcam), and anti-PTEN antibodies (\#9559, Cell Signaling Technology) were incubated overnight at $4{ }^{\circ} \mathrm{C}$ with the membranes. Immune complexes were detected by enhanced chemiluminescence (Cell Signaling Technology).

\section{RNA isolation and quantitative real-time PCR}

The total RNA was isolated from tissues and cell lines using TRIzol reagent (Invitrogen, CA, USA), and exosomal RNA was extracted from plasma and culture medium using the exoRNeasy Midi Kit (Qiagen, Valencia, CA, USA) according to the manufacturer's protocol. The cDNA was synthesized using a high capacity cDNA reverse transcription kit (Thermo Fisher Scientific, Vilnius, Lithuania). Quantitative real-time PCR (qRT-PCR) was conducted with an ABI 7900 system (Applied Biosystems, CA, USA) and SYBR Green assays (TaKaRa Biotechnology, Dalian, China). We chose glyceraldehyde-3-phosphate dehydrogenase (GAPDH) to normalize lncRNA expression levels. The fold change in the expression of lncRNA was calculated with the formula $2^{-\Delta \mathrm{CT}}$. The primer sequences are shown in Additional file 1: Table S1.

\section{The stability of exosomal PTENP1}

To determine the stability of exosomal PTENP1 in plasma, we randomly selected 4 plasma samples, each of which was divided into 8 equal portions and frozen and thawed repeatedly ( 0 cycle, 2 cycles, 4 cycles, and 8 cycles) between $-80{ }^{\circ} \mathrm{C}$ and room temperature, or their incubation at room temperature was prolonged for $0 \mathrm{~h}$, 
$4 \mathrm{~h}, 8 \mathrm{~h}$, and $24 \mathrm{~h}$. The qRT-PCR was used to detect the expression of exosomal PTENP1.

\section{Exosome labeling}

Exosomes from $1.5 \times 10^{6}$ cells were suspended in $100 \mu \mathrm{l}$ of PBS with $1 \mathrm{ml}$ of mixed PKH67 (Sigma, in Diluent C). After 4 min of incubation at room temperature, $2 \mathrm{ml}$ of $0.5 \%$ bovine serum albumin (BSA) was added to terminate exosome labeling, and dyed exosomes were isolated using Exoquick exosome precipitation solution. Exosomes were suspended in $9.6 \mathrm{ml}$ of basal medium, and $250 \mu \mathrm{l}$ was added to the sub-confluent layer of EJ and J82 cells. After incubation for $3 \mathrm{~h}$ at $37{ }^{\circ} \mathrm{C}$, cells were washed and fixed at room temperature. To stain the nuclei, 4',6-diamidino-2-phenylindole (DAPI, Sigma) was added for $10 \mathrm{~min}$, and the stained cells were observed with a fluorescence microscope (Zeiss, LSM700B, Germany). The details of exosome labeling are shown in the Additional file 1.

\section{Cell transfection}

The PTENP1 overexpression plasmid, empty vector (NC) and microRNA-17 (miRNA-17) mimics were synthesized by RiBoBio (Guangzhou, China). The lentiviral vectors containing PTENP1/NC were synthesized by GeneChem (Shanghai, China). Cells were transfected using Lipofectamine 2000 (Invitrogen, Carlsbad, CA, USA) transfection reagent according to the instructions.

\section{The malignant behaviors of bladder cancer cells}

Using EJ and J82 cells, a series of assays were performed to examine the effects of PTENP1 on malignant behaviors, including proliferation, colony formation, invasion, migration, apoptosis, and cell cycle assay. Nuclear and cytosolic fractions of 293A, EJ and J82 cells were separated using the PARIS Kit (Thermo Fisher Scientific, Vilnius, Lithuania). A detailed description of assay conditions is shown in the Additional file 1.

\section{Animal models}

The EJ cell lines $\left(1 \times 10^{7}\right.$ cells in $0.1 \mathrm{ml}$ of PBS) were stably transfected with PTENP1/ NC lentiviral vector and then injected subcutaneously into the right flank of male nude mice (5 weeks old, six mice per group). Tumor growth was examined every two days. The mice were sacrificed after two weeks, and tumor size and weight were measured. To investigate the effect of exosomal PTENP1 in vivo, we only injected EJ cells subcutaneously into the right flank of male nude mice, and 15 days later, when tumors grew to $100 \mathrm{~mm}^{3}$, isolated different exosomes $(10 \mu \mathrm{g})$ from 293A cells transfected with PTENP1/ NC lentiviral vector were then injected into the center of tumor every two days. After 15 days, all mice were sacrificed and tumor from different groups were measured. The animal studies were approved by the Institutional Animal Care and Use Committee of Nanjing Medical University.

\section{Immunohistochemistry (IHC)}

Hematoxylin and eosin (H\&E) staining was utilized to select representative areas. Anti-Ki67 (ab15580, Abcam) and anti-PTEN were applied for IHC. IHC staining was performed according to our previous study [20].

\section{Statistical analysis}

Student's $t$-test or Pearson's $X^{2}$ test was used to access the differences in characteristics between bladder cancer cases and healthy controls. The threshold cycle (CT) value was analyzed using SDS 2.4 software (Applied Biosystems, Foster City, CA, USA). The receiver operating characteristic (ROC) curve was used to reflect the area under the curve (AUC) values for exosomal PTENP1 in plasma. $P<0.05$ was considered statistically significant. All statistical analyses were performed with SAS version 9.4 software (SAS Institute, Inc., Cary, NC, USA).

\section{Results}

\section{Patient characteristics}

The characteristics of the bladder cancer cases and healthy controls are summarized in Table 1 . There were no differences between cases and controls in age, sex, smoking status and pack-years of smoking $(P>0.05)$. The majority of cases were in G1 (42\%) and clinical grade I (82\%), as well as a large proportion of cases with superficial bladder cancer (82\%). The clinical characteristics of paired bladder cancer tissue samples are summarized in Additional file 1: Table S2.

\section{Determination of candidate IncRNAs between bladder cancer tissues and paired normal tissues}

The characteristics of 12 candidate lncRNAs are summarized in Additional file 1: Table S3. As shown in Additional file 1: Figure S1 and Table S4, the expression of H19, SNHG16 and UCA1 was significantly elevated in bladder cancer tissues, while PTENP1 and MEG3 were significantly decreased in bladder cancer tissues compared with adjacent normal tissues $(P<0.05)$.

\section{Expression of plasma exosomal PTENP1 in patients with bladder cancer}

Exosomes from plasma of patients with bladder cancer and healthy controls were isolated and characterized. TEM showed that the sizes of exosomes from cases were consistent with exosomes from healthy controls (50$120 \mathrm{~nm}$, Fig. 1a). Western blots revealed the presence of the exosome markers, TSG101 and CD63 (Fig. 1b). Exosomal RNA was extracted from plasma. Subsequently, we detected the expression of H19, SNHG16, UCA1, PTENP1 
Table 1 The characteristics of the bladder cancer cases and healthy controls

\begin{tabular}{|c|c|c|c|}
\hline Variables & $\begin{array}{l}\text { Case }(n=50) \\
N(\%)\end{array}$ & $\begin{array}{l}\text { Control }(n=60) \\
\mathrm{N}(\%)\end{array}$ & $p^{a}$ \\
\hline Age (years) (mean \pm SD) & $67.0 \pm 9.8$ & $66.2 \pm 10.7$ & 0.694 \\
\hline Sex & & & 0.876 \\
\hline Male & $36(72.0)$ & $44(73.3)$ & \\
\hline Female & $14(28.0)$ & $16(26.7)$ & \\
\hline Smoking status & & & 0.935 \\
\hline Never & $38(76.0)$ & $46(76.7)$ & \\
\hline Ever & $12(24.0)$ & $14(23.3)$ & \\
\hline Pack-years of smoking & & & 0.976 \\
\hline 0 & $38(76.0)$ & $46(76.7)$ & \\
\hline $0-20$ & $3(6.0)$ & $4(6.7)$ & \\
\hline$>20$ & $9(18.0)$ & $10(16.6)$ & \\
\hline \multicolumn{4}{|l|}{ Tumor grade } \\
\hline G1 & $21(42.0)$ & & \\
\hline G2 & $14(28.0)$ & & \\
\hline G3 & $15(30.0)$ & & \\
\hline \multicolumn{4}{|l|}{ Tumor stage } \\
\hline Superficial (pTa-pT1) & $41(82.0)$ & & \\
\hline Invasive (pT2-pT4) & $9(18.0)$ & & \\
\hline \multicolumn{4}{|l|}{ Clinical grade } \\
\hline । & $41(82.0)$ & & \\
\hline$\|$ & $6(12.0)$ & & \\
\hline III & $2(4.0)$ & & \\
\hline IV & $1(2.0)$ & & \\
\hline
\end{tabular}

${ }^{a}$ Student's t-test for age between cases and controls; Two-sided $x^{2}$ for other variables between cases and controls

and MEG3 in exosomes from plasma (Additional file 1: Figure S2A). The expression of PTENP1 in exosomes from cases was significantly lower than that from healthy controls $(P<0.05$, Fig. 1c). Next, we conducted a subgroup analysis by clinicopathologic features (tumor grade, tumor stage and clinical grade) and found that exosomal PTENP1 levels gradually decreased with the deterioration of clinicopathologic features. Moreover, the expression of exosomal PTENP1 in high clinical grade (II + III + IV) was significantly decreased compared with low clinical grade (I) $(P<0.05$, Additional file 1: Figure S2B).

Furthermore, we evaluated the potential usefulness of exosomal PTENP1 as a noninvasive biomarker using generated ROC curves. As shown in Fig. 1d, the AUC of $0.743(95 \% \mathrm{CI}=0.645-0.840)$ was in exosomal PTENP1. The sensitivity and specificity of exosomal PTENP1 to predict the presence of bladder cancer were $65.4 \%$ and $84.2 \%$, respectively. Moreover, we found that exosomal PTENP1 expression did not obviously change along with incubation time at different room temperatures (Fig. 1e), as well as at different freeze-thaw times (Fig. 1f), indicating that PTENP1 is stable in exosomes from plasma. All these results indicated that exosomal PTENP1 might act as a useful biomarker for discriminating patients with bladder cancer from healthy controls.

\section{Effect of PTENP1 on bladder cancer cellular phenotype}

In the current study, we attempted to detect the biological role of PTENP1 in vitro, because we found that the downregulated expression of PTENP1 in bladder cancer tissues and exosomes from bladder cancer plasma. PTENP1-expressing plasmid or NC vector was transfected into EJ and J82 cells. We used qRT-PCR to confirm the expression of PTENP1 (Fig. 2a). Firstly, proliferation of EJ cells was significantly suppressed after PTENP1 overexpression for $48 \mathrm{~h}$ and $72 \mathrm{~h}$, whereas proliferation of $\mathrm{J} 82$ cells was remarkably inhibited after PTENP1 overexpression for $24 \mathrm{~h}, 48 \mathrm{~h}$ and $72 \mathrm{~h}$, as compared with cells transfected with $\mathrm{NC}$ vectors (Fig. $2 \mathrm{~b}$ ). Additionally, we obtained similar results by colony formation assays (Fig. 2c). Moreover, the two cell lines transfected with PTENP1 overexpression for $24 \mathrm{~h}$ revealed remarkably impeded invasive and migratory abilities (Fig. 2d and e). Additionally, flow cytometric analysis showed that PTENP1 overexpression induced apoptosis of EJ and J82 cells (Fig. 2f), as well as an increased percentage of EJ cells in S and G2 phase and an elevated percentage of $J 82$ cells in G2 phase (Fig. 2g).

\section{Exosomal PTENP1 serves as a mediator in intercellular communication}

We explored the existing pattern of extracellular PTENP1. The levels of PTENP1 in the medium were unchanged upon RNase treatment, but were significantly reduced when simultaneously treated with RNase and Triton X-100 (Fig. 3a and Additional file 1: Figure S3A), suggesting that extracellular PTENP1 is packaged by a membrane instead of being directly released. The sizes of exosomes were confirmed by TEM (Fig. 3b), and western blots were performed to show the presence of TSG101 and CD63 (Fig. 3c). We then found that PTENP1 expression was significantly higher in 293A cells than in J82 and EJ cells (Additional file 1: Figure S3B). As expected, exosomal PTENP1 levels had significantly increased in 293A cells compared with $J 82$ and EJ cells (Additional file 1: Figure S3C). Furthermore, PTENP1 levels were enriched by at least three-fold in exosomes compared to those in producer cells (Fig. 3d). Thus, exosomes from 293A cells contained more PTENP1 than those from J82 and EJ cells, in agreement with our findings, and it was overexpressed in healthy controls. Then, exosomes derived from 293A cells were labeled with a green fluorescent marker, PKH67. After recipient cells (J82 and EJ cells) were incubated with labeled exosomes from 293A cells for $3 \mathrm{~h}$, PKH67 was localized in the cytoplasm of recipient cells (Fig. 3e). 
a
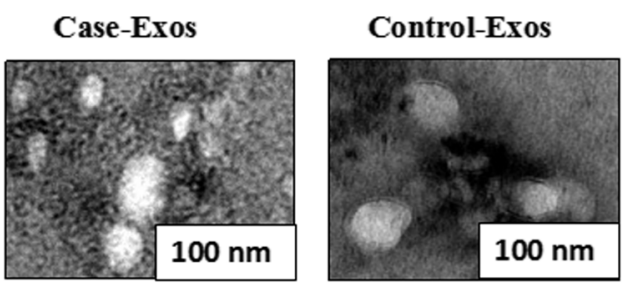

b

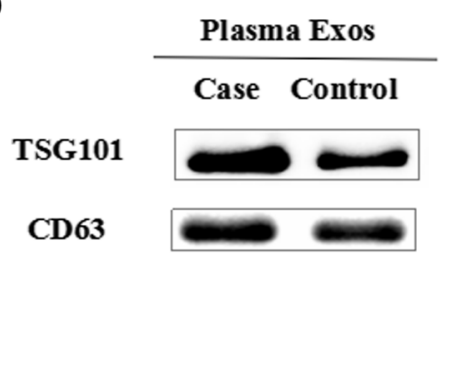

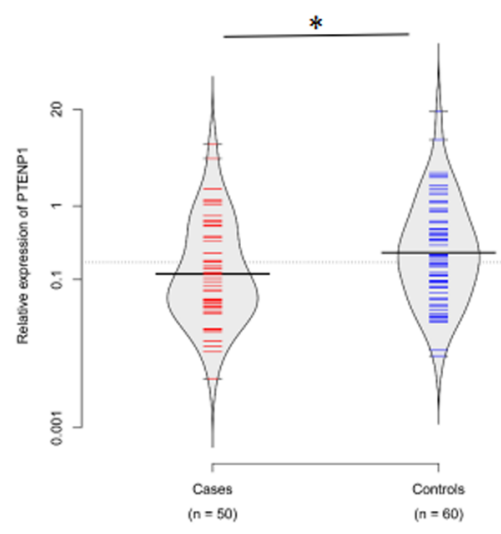

e



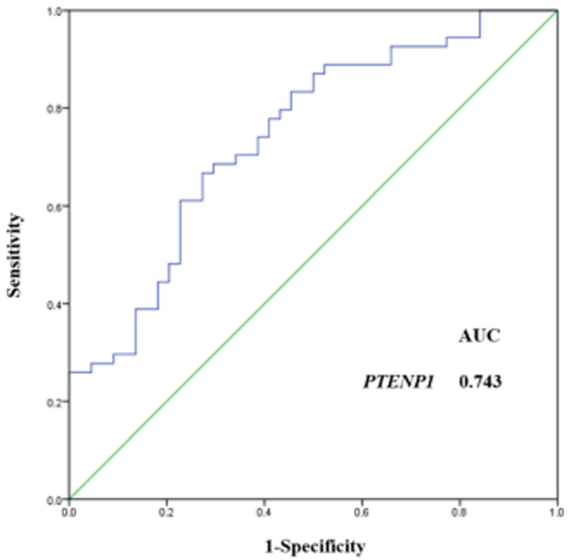

f

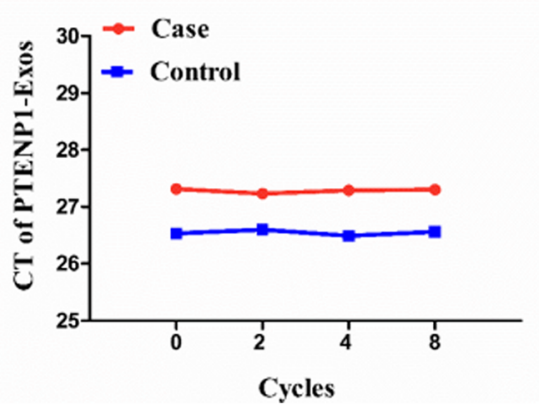

Fig. 1 Expression of plasma exosomal PTENP1 in patients with bladder cancer. Plasma Exosomes (Exos), exos isolated from the plasma of cases and controls. a Micrographs of exos isolated from the plasma of cases (left) and controls (right, bars =100 nm). b Western blots of TSG101 and CD63 in circulating exos. c qRT-PCR detection of PTENP1 in exos from plasma. d ROC curves analysis of exosomal PTENP1 signature. e The expression of exosomal PTENP1 was detected after placing plasma samples at room temperature $0 \mathrm{~h}, 4 \mathrm{~h}, 8 \mathrm{~h}$, and $24 \mathrm{~h}$. $\mathbf{f}$ The expression of exosomal PTENP1 was detected after freezing and thawing plasma samples repeatedly 0 cycle, 2 cycles, 4 cycles and 8 cycles. Results are presented as mean \pm SD. ${ }^{*} P<0.05$

\section{Effect of exosomal PTENP1 on bladder cancer cellular phenotype}

Exosomes are known to play important roles in cell-cell communication, which may change the physiological function of the recipient cells by bioactive factors, including IncRNAs [21]. The frequent silencing of PTENP1 in patients with bladder cancer and bladder cancer cell lines but not in controls indicates that PTENP1 may act as a tumor suppressor. As described in this study, we have found that the transfer of PTENP1 from 293A cells into EJ and J82 cells occurs via exosomes. We then predicted that exosomal PTENP1 derived from 293A cells could change the biological function of EJ and J82 cells. However, exosomes contain diverse cargoes, such as transcriptional regulators, various RNA species, DNA and lipids [12]. To establish functions of exosomal PTENP1, we isolated exosomes from 293A cells transfected with PTENP1-expressing plasmid or NC vector, namely PTENP1-Exos or NC-Exos. We added PTENP1-Exos or NC-Exos at various concentrations $(0,20,40,60,80$, or $100 \mu \mathrm{g} / \mathrm{ml})$ to EJ and J82 cells for $24 \mathrm{~h}$. The results showed that 


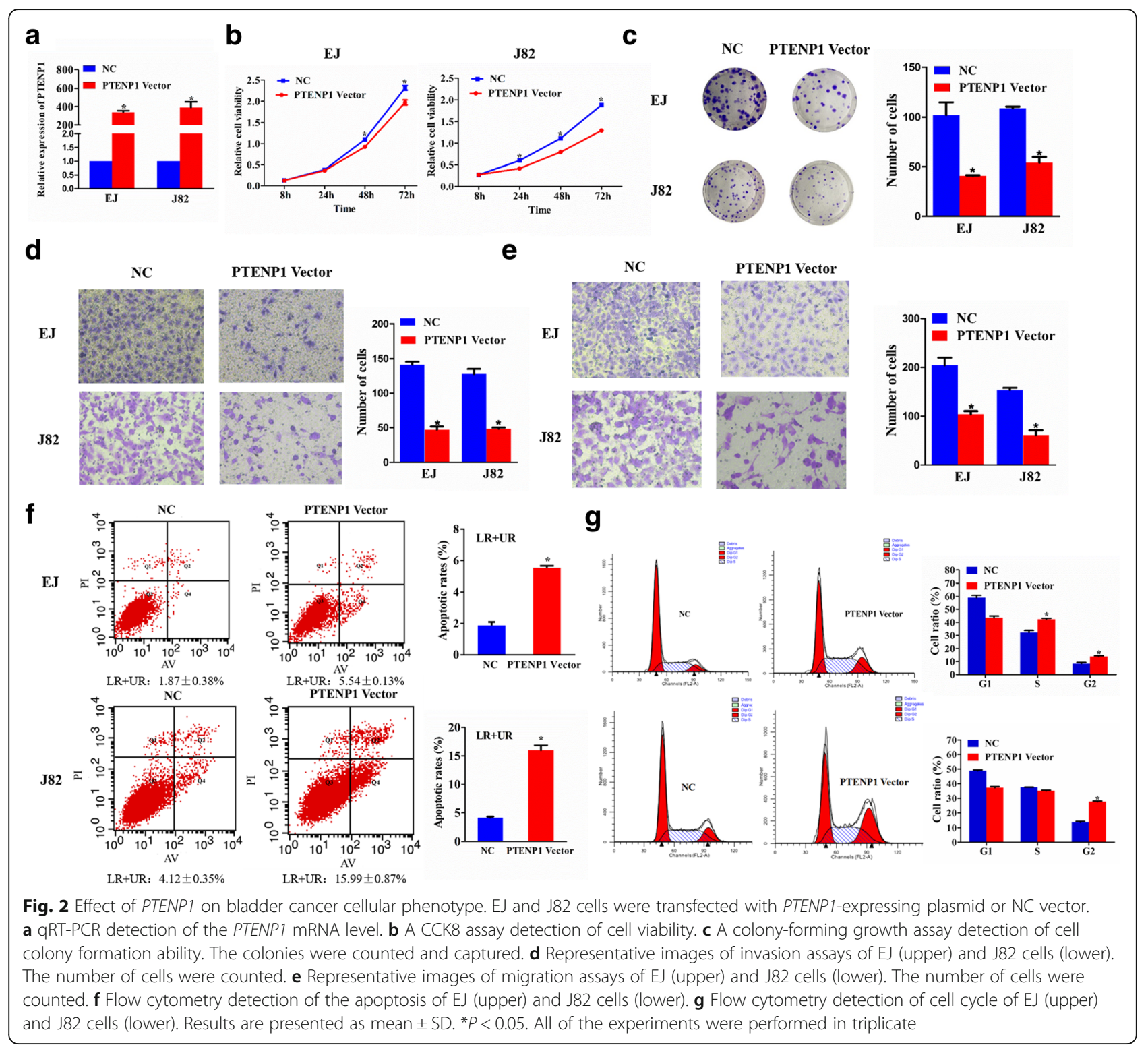

PTENP1-Exos could significantly reduce proliferation of $E J$ and J82 cells in a concentration-dependent fashion when the concentration of exosomes was higher than $60 \mu \mathrm{g} / \mathrm{ml}$ (Fig. 4a). Thus, all bladder cancer cellular phenotypes were examined with the following treatment of $60 \mu \mathrm{g} / \mathrm{ml}$ of exosomes for $24 \mathrm{~h}$ in the further study. The levels of PTENP1 in EJ and J82 cells exposed to PTEN$P 1$-Exos were significantly increased compared to EJ and J82 cells exposed to NC-Exos for $24 \mathrm{~h}$ (Fig. 4b). In addition, we used colony formation assays to obtain similar results (Fig. 4c). Furthermore, PTENP1-Exos inhibited the invasive and migratory abilities of EJ and J82 cells (Fig. 4d and e). Additionally, PTENP1-Exos increased the numbers of apoptotic EJ and J82 cells (Fig. 4f), as well as promoted cycle arrest of EJ cells at the S and G2 phase and stimulated cycle arrest of J82 cells at the G2 phase (Fig. 4g). Thus, after exposure to PTENP1-Exos derived from 293A cells, PTENP1 was overexpressed and the biologically malignant behavior was inhibited in recipient EJ and J82 cells.

\section{Effect of PTENP1 overexpression and exosomal PTENP1 on tumor in vivo}

To further explore the role of PTENP1 overexpression and exosomal PTENP1 in bladder cancer in vivo, we injected EJ cells with PTENP1/ NC lentiviral vector or PTENP1/NC Exos derived from 293A cells transfected with PTENP1/ NC lentiviral vector into nude mice. In line with in vitro analysis, PTENP1 overexpression significantly decreased the mean tumor weight and average tumor volume (Fig. 5a-d) as compared with NC group. As shown in Fig. $5 \mathrm{a}-\mathrm{d}$, the tumor tissues of nude mice injected with 
a



c



e

J82
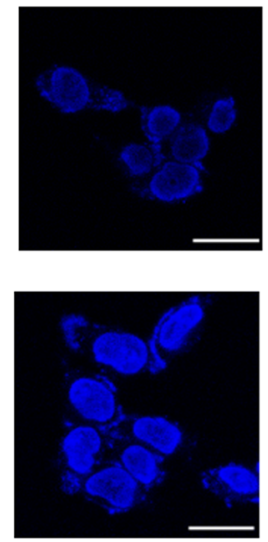

b

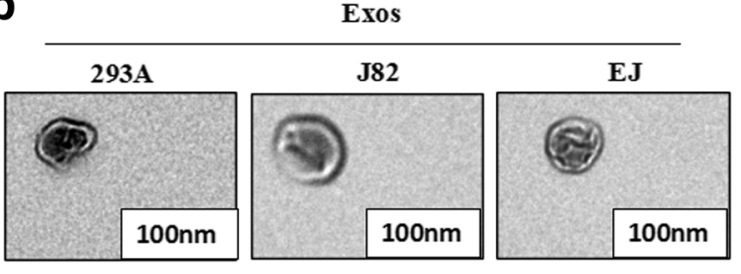

d



PKH67


DAPI/PKH67


Fig. 3 Exosomal PTENP1 serve as a mediator in intercellular communication. Exosomes (Exos) isolated from the medium of $293 \mathrm{~A}$, J82 and EJ cells. a qRT-PCR detection of the normalized expression of PTENP1 in the medium of 293A, J82 and EJ cells treated with RNase $(2 \mu \mathrm{g} / \mathrm{ml})$ alone or combined with Triton X-100 (0.1\%) for 20 min. b Micrographs of exos isolated from 293A (left), J82 (middle) and EJ cells (right, bars =100 nm). c Western blots of TSG101 and CD63 in exos of cell lines. d qRT-PCR detection of the fold change of PTENP1 between exos of 293A, J82 and EJ and their producer cells. e Exos of 293A cells were labeled with PKH67; green represents PKH67, and blue represents nuclear DNA staining by DAPI. J82 and EJ cells were incubated with exos derived from $293 \mathrm{~A}$ cells for $3 \mathrm{~h}$. Results are presented as mean \pm SD. ${ }^{*} P<0.05$. All of the experiments were performed in triplicate

PTENP1-Exos also attenuated tumor size and weight. The tumor tissues of mice inoculated in PTENP1 vector and PTENP1-Exos presented an increased expression of PTENP1 (Fig. 5e). Moreover, H\&E staining and immunohistochemistry for proliferation marker Ki67 was performed to investigate Ki67 expression. As shown in Fig. 5f, Ki67 expression was significantly reduced in PTENP1 overexpression and PTENP1-Exos models.

\section{Exosomal PTENP1 regulates PTEN expression via miR-17} It is well known that PTENP1 is a processed pseudogene of PTEN, and exerts high degree of homology to PTEN [22]. Thus, we added PTENP1-expressing plasmid/NC vector or PTENP1-Exos/NC-Exos to EJ and J82 cells, and detected PTEN expression. The results revealed that overexpression of PTENP1 could increase PTEN expression levels (Fig. 6a). Interestingly, PTENP1-Exos also elevated the expression of PTEN in bladder cancer cells by western blot (Fig. 6b). Furthermore, a substantial increase of PTEN expression was identified in vivo with PTENP1 overexpression and PTENP1-Exos (Fig. 5e and $\mathrm{f}$ ). As described in this study, we have demonstrated that extracellular PTENP1 was mainly wrapped by exosomes. These data prompted us to focus on the molecular mechanisms of exosomal PTENP1. Many studies have reported that PTENP1 can competitively bind miR-17 to modulate PTEN expression [19, 23, 24]. Thus, we sought to investigate whether exosomal PTENP1 could regulate PTEN levels by competitively binding to miR-17. The bioinformatics analysis revealed that 


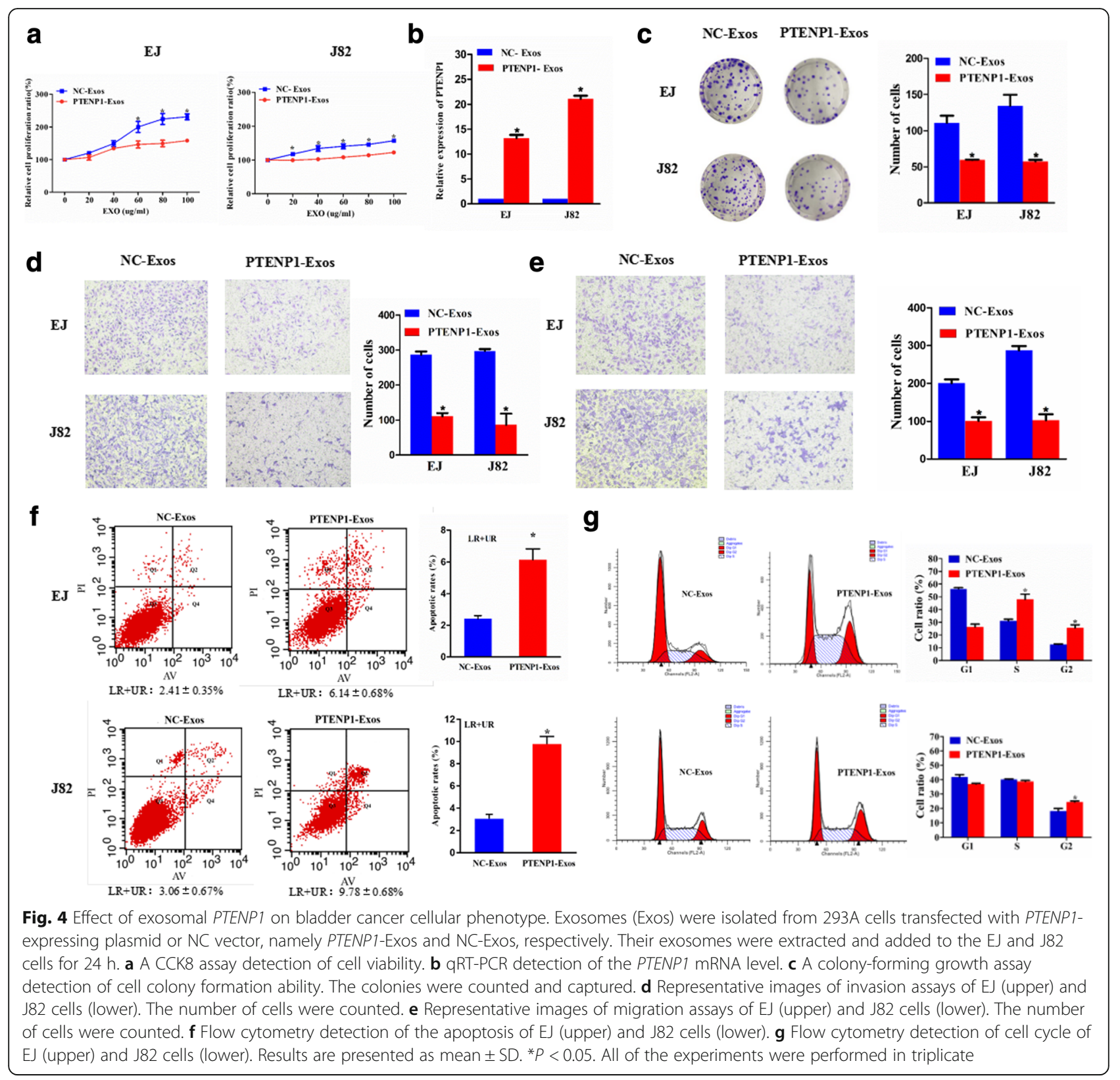

exosomal PTENP1 may exert its antitumor effect by regulating the expression of PTEN (Fig. 6c). As shown in Fig. 6d, miR-17 mimic reduced the expression of PTEN, whereas exosomal PTENP1 substantially eliminated this effect.

\section{Discussion}

Bladder cancer is a urinary system malignancy that is common worldwide, and its etiology is complex [2]. We identified that PTENP1 was mainly in exosomes, and found that PTENP1 was downregulated in bladder cancer tissues and plasma. Additionally, this study showed that normal cells could package PTENP1 into exosomes and secrete it into bladder cancer cells, which significantly suppressed malignant behaviors of bladder cancer cells by decreasing the ability to proliferate, invade and migrate. Exosomal PTENP1 also inhibited the growth of tumor in vivo. Thus, exosomal PTENP1 could be a potential candidate of bladder cancer detection. In addition, we confirmed that exosomal PTENP1 protected PTEN and suppressed biological malignant behavior of bladder cancer by sponging miR-17 (Fig. 7).

Several studies have revealed that dysregulated lncRNAs were expressed in many cancers, including bladder cancer $[25,26]$. In the present study, we enrolled a total of 12 major lncRNAs with important roles in multiple tumors until December 2016, among which Kcnq1ot1 and PTENP1 have not been investigated in bladder cancer. 




Fig. 5 Effect of PTENP1 overexpression and exosomal PTENP1 on tumor in vivo. PTENP1/ NC lentiviral vector was transfected into EJ cells, namely PTENP1 vector and NC, respectively. Exosomes (Exos) were isolated from 293A cells transfected with PTENP1/ NC lentiviral vector, namely PTENP1Exos and NC-EXOs, respectively. a. Burdened nude mice inoculated in NC, PTENP1-EXOS, PTENP1 vector and NC-Exos. Red arrows show position of tumor. b. The xenografts from nude mice inoculated in NC, PTENP1-ExOS, PTENP1 vector and NC-Exos. c. The tumor volumes were measured every two days after injection. $\mathbf{d}$. The tumor weights in nude mice at the 15 day were determined. e. Detection of PTENP1 and PTEN expression in tumor tissues of nude mice treated with NC, PTENP1 vector, PTENP1-Exos and NC-ExOs by qRT-PCR. f. H\&E stained images and immunohistochemistry analysis of ki67 and PTEN expression in tumor tissues. Results are presented as mean \pm SD. ${ }^{*} P<0.05$

This study showed that H19, SNHG16, UCA1, PTENP1 and $M E G 3$ were aberrantly expressed in bladder cancer tissues. To date, accumulated evidences have shown that exosomal lncRNAs reflect the physiological condition of the donor cells, and being captured by recipient cells induces a series of cellular responses [27, 28]. Additionally, exosomal IncRNAs have been reported as potential cancer biomarkers for patients with bladder, gastric, colorectal and cervical cancer [28-31], suggesting that exosomal lncRNAs can be potential biomarkers for identifying cancers.

Here, we performed TEM to reveal the shapes and size of exosomes from plasma. Exosomes were also verified using the exosome markers TSG101 and CD63 [32, 33]. We then detected the expression levels of H19, SNHG16, UCA1, PTENP1 and MEG3 in exosomes from plasma. Interestingly, we found that exosomal PTENP1 levels were downregulated in patients with bladder cancer, and lower exosomal PTENP1 expression was associated with higher tumor and clinical grade, as well as more advanced stage. After repeated freeze-thaw cycles and incubation at room temperature, there was no significant change in the expression of exosomal PTENP1, suggesting that PTENP1 is stable in the exosomes of plasma. Although our ROC analyses revealed that exosomal PTENP1 could be useful in detecting bladder cancer, we needed to further analyze large samples to evaluate its diagnostic accuracy. Accumulating evidences have shown that lncRNAs play critical roles in controlling a variety of biochemical cellular processes, including proliferation, invasion, migration and apoptosis [34, 35]. These results led us to further examine the putative tumor suppressor function of PTENP1 in human bladder cancer cells. We carried out a series of assays and found that overexpression of PTENP1 reduced cell proliferation, 
EJ

a

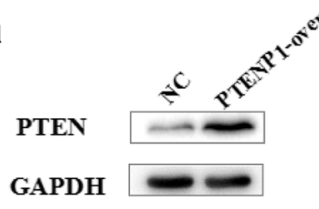

b

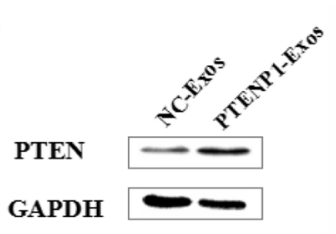

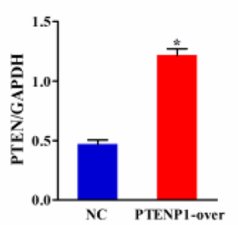

EJ

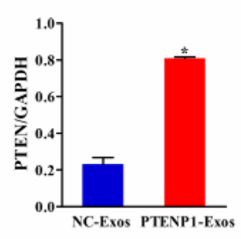

J82

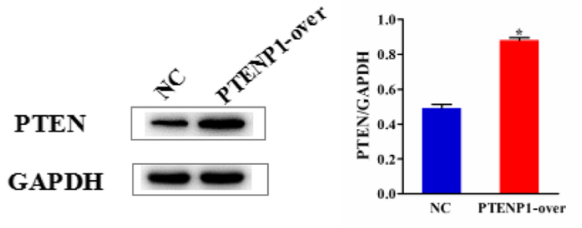

J82

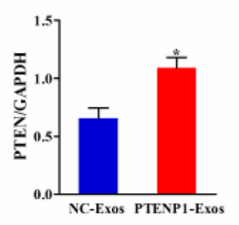

C

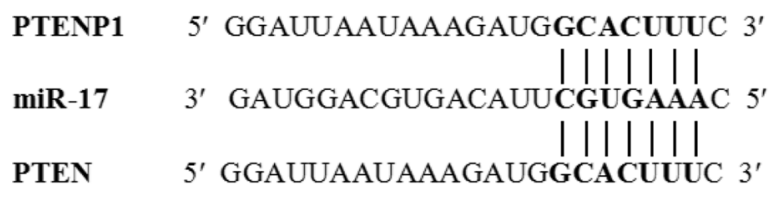

d

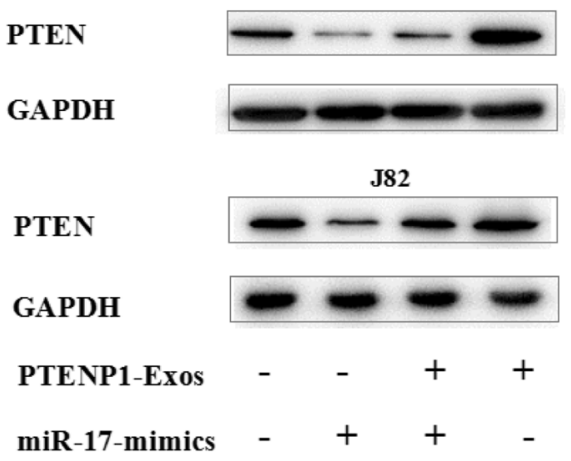

Fig. 6 EXosomal PTENP1 regulates PTEN expression via miR-17. a. Western blots of PTEN in EJ and J82 cells were transfected with PTENP1-expressing plasmid or NC vector. b. Western blots of PTEN in EJ and $J 82$ cells were treated with PTENP1-ExOs or NC-Exos. c. Putative miR-17 binding sequence in the 3'-UTR of PTEN mRNA. d. Western blots of PTEN in EJ and J82 cells with PTENP1-Exos and/ or miR-17 mimics. Results are presented as mean \pm SD. ${ }^{*} P<0.05$. All of the experiments were performed in triplicate

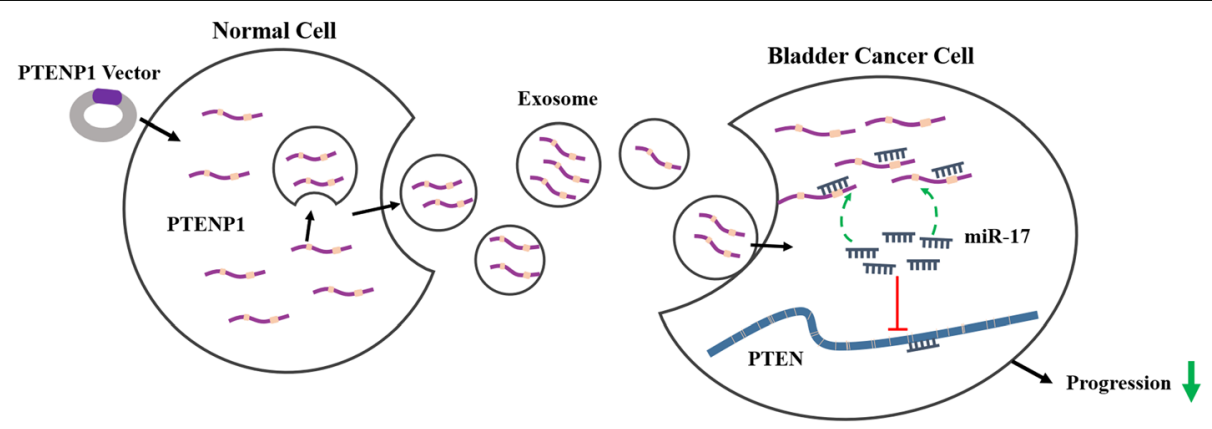

Fig. 7 Schematic diagram of exosomal PTENP1-mediated bladder cancer progression. Exosomal PTENP1 derived from normal cells transfected with PTENP1 vector enhance PTENP1 expression of bladder cancer cells. Exosomal PTENP1 suppresses the progression of bladder cancer by acting as a ceRNA to competitively bind to miR-17 and regulate PTEN expression 
migration and invasion, as well as induced cell apoptosis. Furthermore, previous studies have reported that the differentiation level of bladder cancer is assessed routinely in the clinic, poorly differentiated tumors generally exhibiting the worst prognoses [36, 37]. Therefore, we chose EJ (poorly differentiated) and J82 (well-differentiated) cell lines to evaluate the effect of PTENP1 on cellular phenotypes. Interestingly, we found that PTENP1 decreased the well-differentiated J82 cell viability was more obvious than poorly differentiated EJ cells in $24 \mathrm{~h}, 48 \mathrm{~h}$ and $72 \mathrm{~h}$ time points (Additional file 1: Figure S4A). Meanwhile, animal experiments also supported the tumor suppressor function of PTENP1 in bladder cancer.

As described in our data, we have identified that PTENP1 was mainly secreted from cells via exosomes. Exosomes are extracellular vesicles of endocytic origin and incorporate into components of donor cells, including signaling proteins, various RNA species (including lncRNA and other species), transcriptional regulators, DNA and lipids that can be taken up into neighboring or distant cell types to regulate the function of recipient cells [21, 38, 39]. The roles of exosomal lncRNAs have been intensely investigated in cancers [40, 41]. For example, exosomes released from sunitinib-resistant cells transferred lncARSR to sensitive cells, which induced sunitinib resistance in renal cancer [42]. Exosomal linc-VLDL derived from hepatocellular cancer cells exposed to sorafenib and doxorubicin was transferred into recipient tumor cells, which increased linc-VLDL expression and decreased chemotherapy-induced cell death in recipient cells [43]. In the present effort, we discovered that levels of PTENP1 were increased in normal cells and their exosomes, and its expression in exosomes was approximately three times greater than that in producer cells. Similar to other reports $[44,45]$, fluorescence microscopy revealed that exosomes derived from normal cells that were labeled with PKH67 could transfer into bladder cancer cells. These findings indicate the possibility that 293A cells secrete exosomal PTENP1 for transferring to the surrounding bladder cancer cells. Although normal cells exposed to exosomal lncRNAs secreted from cancer cells have been widely studied, there have been few reports describing exosomal lncRNAs released from normal cells that are transferred into cancer cells. Our results showed that PTENP1-Exos from 293A cells enhanced PTENP1 expression and suppressed proliferation, migration and invasion of bladder cancer cells; in addition, it induced apoptosis in bladder cancer cells. However, exosomal PTENP1 was not significantly reduced the viability of well-differentiated $J 82$ cells compared to poorly differentiated EJ cells (Additional file 1: Figure S4B). This could be due to the variety of cargoes in exosomes, indicating that we will comprehensively evaluate antitumor effects of intercellular/exosomal
PTENP1 between well-differentiated 182 cells and poorly differentiated EJ cells. Consistent with our previous findings in vitro, exosomal PTENP1 also repressed tumor growth in vivo. Hence, our findings suggest that PTENP1 is directly transferred from normal cells to bladder cancer cells via exosomes and regulate the biological functions of bladder cancer in vitro and in vivo.

Previous studies have reported that PTENP1 is a pseudogene-expressed lncRNA located at 9p13.3, which competitively binds miRNAs to modulate PTEN expression by serving as a competing endogenous RNA (ceRNA) in several cancers [46-48]. Thus, we speculated that PTENP1 may act as a ceRNA, participating in tumorigenesis of bladder cancer. Our findings revealed that exosomal PTENP1 could regulate PTEN levels by competing for the effect of miR-17. Our results showed that PTENP1 and exosomal PTENP1 were also enriched in the nucleus of cell lines, suggesting that it may also physically localizes to the PTEN promoter, where it attracts some chromatin modification enzymes that cause epigenetic silencing (Additional file 1: Figure S5A and B). Therefore, further studies should be performed to comprehensively investigate the biology of intercellular PTENP1 and exosomal PTENP1 involved in bladder cancer development and progression.

\section{Conclusion}

In conclusion, our results indicated that exosomal PTENP1 was a promising novel biomarker for diagnosis of bladder cancer. In addition, normal cells released exosomes containing PTENP1, and that exosomal PTENP1 was transported from normal cells to bladder cancer cells, and exogenous PTENP1 relieved the malignant phenotype of bladder cancer cells both in vitro and in vivo. Furthermore, exosomal PTENP1 may act as a miR-17 decoy to regulate PTEN and suppress bladder cancer progression. Together, our results revealed that exosomal PTENP1 serves as a mediator in cell-cell communication during carcinogenesis of bladder cancer.

\section{Additional file}

Additional file 1: Table S1. Primer sequences in the study. Table S2. Clinical characteristics of paired bladder cancer tissue samples. Table S3. The characteristics of candidate IncRNAs until December 2016. Table S4. The relative expression of 12 candidate IncRNAs in paired bladder cancer tissues. Figure S1-S5 (online). Supplementary materials (online). (DOC 2980 kb)

Abbreviations

AUC: Area under curve; IncRNA: Long non-coding RNA; PTEN: Phosphatase and tensin homolog; PTENP1: Phosphatase and tensin homolog pseudogene 1; ROC: Receiver operating characteristic curve 


\section{Acknowledgments}

This study was partly supported by National Natural Science Foundation of China (81473050 and 81703307), Jiangsu Provincial "333" project, Beijing Municipal Administration of Hospitals' Youth Programme (QML20150101)، the Priority Academic Program Development of Jiangsu (Public Health and Preventive Medicine), Natural Science Foundation of Jiangsu Province (BK20151603), Nanjing Science and Technology Program (201605009) and Collaborative Innovation Center for Cancer Personalized Medicine.

\section{Availability of data and materials}

Not applicable.

\section{Authors' contributions}

$Z Z, Q J, Y L, W M, D M, Z R$ and $C H$ designed and performed the research. XW. WW, LQ, QC, and YL recruited samples. DM, ZR, and XW analyzed and interpreted data. $Z R, W X$ and $L$ performed the experiments. $Z R, D M, W X$, and XW wrote the draft manuscript. All authors contributed to the writing and reviewing of the manuscript, and approved the final manuscript for submission.

\section{Ethics approval and consent to participate}

The research was approved by the Ethics Committee of Nanjing Medical University.

\section{Consent for publication}

All authors consented for publication.

\section{Competing interests}

The authors declare that they have no competing interests.

\section{Publisher's Note}

Springer Nature remains neutral with regard to jurisdictional claims in published maps and institutional affiliations.

\begin{abstract}
Author details
${ }^{1}$ Department of Environmental Genomics, School of Public Health, Jiangsu Key Laboratory of Cancer Biomarkers, Prevention and Treatment, Collaborative Innovation Center for Cancer Personalized Medicine, Nanjing Medical University, 101 Longmian Avenue, Jiangning District, Nanjing 211166, China. ${ }^{2}$ Department of Genetic Toxicology, The Key Laboratory of Modern Toxicology of Ministry of Education, School of Public Health, Nanjing Medical University, Nanjing, China. ${ }^{3}$ Department of Biostatistics, School of Public Health, Nanjing Medical University, Nanjing, China. ${ }^{4}$ Department of Urology, Yizheng Hospital, Drum Tower Hospital Group of Nanjing, Yizheng, People's Republic of China. ${ }^{5}$ Department of Urology, Beijing Friendship Hospital affiliated to Capital Medical University, Beijing, People's Republic of China. ${ }^{6}$ Department of Urology, The First Affiliated Hospital of Nanjing Medical University, Nanjing, China. ${ }^{7}$ Department of Urology, Jiangsu Province Hospital of TCM, 155 Hanzhong Road, Nanjing 210029, People's Republic of China. ${ }^{8}$ Department of Integrated Traditional Chinese and Western Medicine Tumor Research Lab, Nanjing, People's Republic of China. ${ }^{9}$ Department of General Surgery, Yizheng Hospital, Drum Tower Hospital Group of Nanjing, 1 Huannan Road, Yizheng 211900, People's Republic of China.
\end{abstract}

Received: 7 February 2018 Accepted: 16 August 2018

Published online: 03 October 2018

\section{References}

1. Torre LA, Bray F, Siegel RL, Ferlay J, Lortet-Tieulent J, Jemal A. Global cancer statistics, 2012. CA Cancer J Clin. 2015;65:87-108.

2. Ploeg M, Aben KK, Kiemeney LA. The present and future burden of urinary bladder cancer in the world. World J Urol. 2009;27:289-93.

3. Siegel RL, Miller KD, Jemal A. Cancer statistics, 2017. CA Cancer J Clin. 2017; 67:7-30.

4. Chen W, Zheng R, Baade PD, Zhang S, Zeng H, Bray F, Jemal A, Yu XQ, He J. Cancer statistics in China, 2015. CA Cancer J Clin. 2016;66:115-32.

5. Pang C, Guan Y, Li H, Chen W, Zhu G. Urologic cancer in China. Jpn J Clin Oncol. 2016;46:497-501.

6. Mitra SA, Mitra AP, Triche TJ. A central role for long non-coding RNA in cancer. Front Genet. 2012;3
7. Chi HC, Tsai CY, Tsai MM, Yeh CT, Lin KH. Roles of long noncoding RNAs in recurrence and metastasis of radiotherapy-resistant Cancer stem cells. Int J Mol Sci. 2017;18

8. Yoshimoto R, Mayeda A, Yoshida M, Nakagawa S. MALAT1 long non-coding RNA in cancer. Biochim Biophys Acta. 2016;(1):192-9.

9. Taheri M, Omrani MD, Ghafouri-Fard S. Long non-coding RNA expression in bladder cancer. Biophys Rev. 2017;8:017-0379.

10. Yang G, Wang Y, Zeng Y, Gao GF, Liang X, Zhou M, Wan X, Yu S, Jiang Y, Naghavi M, et al. Rapid health transition in China, 1990-2010: findings from the global burden of disease study 2010. Lancet. 2013;381:1987-2015.

11. Ricciuti B, Mencaroni C, Paglialunga L, Paciullo F, Crino L, Chiari R, Metro G. Long noncoding RNAs: new insights into non-small cell lung cancer biology, diagnosis and therapy. Med Oncol. 2016;33:016-0731.

12. Xu R, Greening DW, Zhu HJ, Takahashi N, Simpson RJ. Extracellular vesicle isolation and characterization: toward clinical application. J Clin Invest. 2016; 126:1152-62.

13. Abels ER, Breakefield XO. Introduction to extracellular vesicles: biogenesis, RNA cargo selection, content, release, and uptake. Cell Mol Neurobiol. 2016; 36(3):301-12. https://doi.org/10.1007/s10571-016-0366-z. Epub 2016 Apr 6.

14. Choi DS, Kim DK, Kim YK, Gho YS. Proteomics, transcriptomics and lipidomics of exosomes and ectosomes. Proteomics. 2013;13:1554-71.

15. Kowal J, Tkach M, Thery C. Biogenesis and secretion of exosomes. Curr Opin Cell Biol. 2014:29:116-25.

16. Revenfeld AL, Baek R, Nielsen MH, Stensballe A, Varming K, Jorgensen M. Diagnostic and prognostic potential of extracellular vesicles in peripheral blood. Clin Ther. 2014;36:830-46.

17. Mohankumar S, Patel T. Extracellular vesicle long noncoding RNA as potential biomarkers of liver cancer. Brief Funct Genomics. 2016;15:249-56.

18. Johnsson P, Ackley A, Vidarsdottir L, Lui WO, Corcoran M, Grander D, Morris KV. A pseudogene long-noncoding-RNA network regulates PTEN transcription and translation in human cells. Nat Struct Mol Biol. 2013;20:440-6.

19. Chen CL, Tseng YW, Wu JC, Chen GY, Lin KC, Hwang SM, Hu YC. Suppression of hepatocellular carcinoma by baculovirus-mediated expression of long noncoding RNA PTENP1 and MicroRNA regulation. Biomaterials. 2015;44:71-81.

20. Wang S, Wu S, Zhu H, Ding B, Cai Y, Ni J, Wu Q, Meng Q, Zhang X, Zhang C, et al. PSCA rs2294008 polymorphism contributes to the decreased risk for cervical cancer in a Chinese population. Sci Rep. 2016;6

21. Colombo M, Raposo G, Thery C. Biogenesis, secretion, and intercellular interactions of exosomes and other extracellular vesicles. Annu Rev Cell Dev Biol. 2014;30:255-89.

22. Poliseno L, Salmena L, Zhang J, Carver B, Haveman WJ, Pandolfi PP. A coding-independent function of gene and pseudogene mRNAs regulates tumour biology. Nature. 2010;465:1033-8.

23. Rao E, Jiang C, Ji M, Huang $X$, labal J, Lenz G, Wright G, Staudt LM, Zhao Y, McKeithan TW, et al. The miRNA-17 approximately 92 cluster mediates chemoresistance and enhances tumor growth in mantle cell lymphoma via PI3K/AKT pathway activation. Leukemia. 2012;26:1064-72.

24. Poliseno L, Pandolfi PP. PTEN ceRNA networks in human cancer. Methods. 2015:78:41-50.

25. Martens-Uzunova ES, Bottcher R, Croce CM, Jenster G, Visakorpi T, Calin GA. Long noncoding RNA in prostate, bladder, and kidney cancer. Eur Urol. 2014;65:1140-51.

26. Peter S, Borkowska E, Drayton RM, Rakhit CP, Noon A, Chen W, Catto JW. Identification of differentially expressed long noncoding RNAs in bladder cancer. Clin Cancer Res. 2014;20:5311-21.

27. Savelyeva AV, Baryakin DN, Chikova ED, Kuligina EV, Richter VA, Semenov DV. Vesicular and extra-vesicular RNAs of human blood plasma. Adv Exp Med Biol. 2016:924:117-9.

28. Dong L, Lin W, Qi P, Xu MD, Wu X, Ni S, Huang D, Weng WW, Tan C, Sheng $W$, et al. Circulating long RNAs in serum extracellular vesicles: their characterization and potential application as biomarkers for diagnosis of colorectal Cancer. Cancer Epidemiol Biomark Prev. 2016;25:1158-66.

29. Li Q, Shao Y, Zhang X, Zheng T, Miao M, Qin L, Wang B, Ye G, Xiao B, Guo J. Plasma long noncoding RNA protected by exosomes as a potential stable biomarker for gastric cancer. Tumour Biol. 2015;36:2007-12.

30. Zhang J, Liu SC, Luo XH, Tao GX, Guan M, Yuan H, Hu DK. Exosomal long noncoding RNAs are differentially expressed in the Cervicovaginal lavage samples of cervical Cancer patients. J Clin Lab Anal. 2016;30:1116-21.

31. Berrondo C, Flax J, Kucherov V, Siebert A, Osinski T, Rosenberg A, Fucile C, Richheimer S, Beckham CJ. Expression of the long non-coding RNA HOTAIR correlates with disease progression in bladder Cancer and is contained in bladder Cancer patient urinary exosomes. PLoS One. 2016;11 
32. Chen C, Luo F, Liu X, Lu L, Xu H, Yang Q, Xue J, Shi L, Li J, Zhang A, Liu Q. NF-kB-regulated exosomal miR-155 promotes the inflammation associated with arsenite carcinogenesis. Cancer Lett. 2017;388:21-33.

33. Fang T, Lv H, Lv G, Li T, Wang C, Han Q, Yu L, Su B, Guo L, Huang S, et al. Tumor-derived exosomal miR-1247-3p induces cancer-associated fibroblast activation to foster lung metastasis of liver cancer. Nat Commun. 2018;9: 017-02583.

34. Batista PJ, Chang HY. Long noncoding RNAs: cellular address codes in development and disease. Cell. 2013;152:1298-307.

35. Zhu Y, Yu M, Li Z, Kong C, Bi J, Li J, Gao Z. ncRAN, a newly identified long noncoding RNA, enhances human bladder tumor growth, invasion, and survival. Urology. 2011;77:13.

36. Ben-Porath I, Thomson MW, Carey VJ, Ge R, Bell GW, Regev A, Weinberg RA. An embryonic stem cell-like gene expression signature in poorly differentiated aggressive human tumors. Nat Genet. 2008:40:499-507.

37. Sgambato A, Migaldi M, Faraglia B, Garagnani L, Romano G, De Gaetani C, Ferrari P, Capelli G, Trentini GP, Cittadini A. Loss of P27Kip1 expression correlates with tumor grade and with reduced disease-free survival in primary superficial bladder cancers. Cancer Res. 1999;59:3245-50.

38. Simpson RJ, Lim JW, Moritz RL, Mathivanan S. Exosomes: proteomic insights and diagnostic potential. Expert Rev Proteomics. 2009;6:267-83.

39. Rak J, Guha A. Extracellular vesicles--vehicles that spread cancer genes. Bioessays. 2012;34:489-97.

40. Conigliaro A, Costa V, Lo Dico A, Saieva L, Buccheri S, Dieli F, Manno M, Raccosta S, Mancone C, Tripodi M, et al. CD90+ liver cancer cells modulate endothelial cell phenotype through the release of exosomes containing H19 IncRNA. Mol Cancer. 2015;14:015-0426.

41. Xue M, Chen W, Xiang A, Wang R, Chen H, Pan J, Pang H, An H, Wang X, Hou H, Li X. Hypoxic exosomes facilitate bladder tumor growth and development through transferring long non-coding RNA-UCA1. Mol Cancer. 2017;16:017-0714

42. Qu L, Ding J, Chen C, Wu ZJ, Liu B, Gao Y, Chen W, Liu F, Sun W, Li XF, et al. Exosome-transmitted IncARSR promotes Sunitinib resistance in renal Cancer by acting as a competing endogenous RNA. Cancer Cell. 2016;29:653-68.

43. Takahashi K, Yan IK, Wood J, Haga H, Patel T. Involvement of extracellular vesicle long noncoding RNA (linc-VLDLR) in tumor cell responses to chemotherapy. Mol Cancer Res. 2014;12:1377-87.

44. Umezu T, Ohyashiki K, Kuroda M, Ohyashiki JH. Leukemia cell to endothelial cell communication via exosomal miRNAs. Oncogene. 2013:32:2747-55.

45. Xu Y, Luo F, Liu Y, Shi L, Lu X, Xu W, Liu Q. Exosomal miR-21 derived from arsenite-transformed human bronchial epithelial cells promotes cell proliferation associated with arsenite carcinogenesis. Arch Toxicol. 2015;89: 1071-82.

46. Yu G, Yao W, Gumireddy K, Li A, Wang J, Xiao W, Chen K, Xiao H, Li H, Tang $K$, et al. Pseudogene PTENP1 functions as a competing endogenous RNA to suppress clear-cell renal cell carcinoma progression. Mol Cancer Ther. 2014; 13:3086-97.

47. Gao L, Ren W, Zhang L, Li S, Kong X, Zhang H, Dong J, Cai G, Jin C, Zheng D, Zhi K. PTENp1, a natural sponge of miR-21, mediates PTEN expression to inhibit the proliferation of oral squamous cell carcinoma. Mol Carcinog. 2017;56:1322-34

48. Guo X, Deng L, Deng K, Wang H, Shan T, Zhou H, Liang Z, Xia J, Li C. Pseudogene PTENP1 suppresses gastric Cancer progression by modulating PTEN. Anti Cancer Agents Med Chem. 2016;16:456-64.

\section{Ready to submit your research? Choose BMC and benefit from:}

- fast, convenient online submission

- thorough peer review by experienced researchers in your field

- rapid publication on acceptance

- support for research data, including large and complex data types

- gold Open Access which fosters wider collaboration and increased citations

- maximum visibility for your research: over $100 \mathrm{M}$ website views per year

At $\mathrm{BMC}$, research is always in progress.

Learn more biomedcentral.com/submissions 